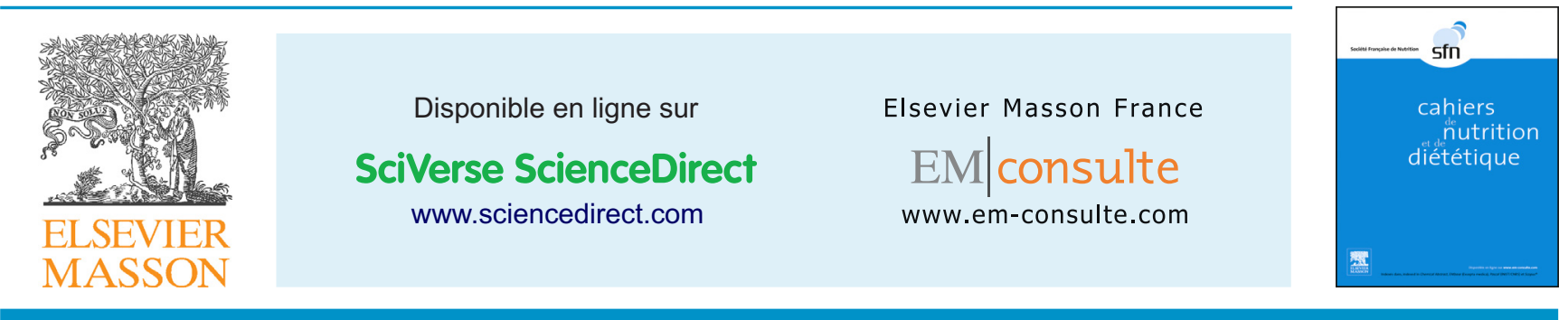

HISTOIRE

\title{
Détection isotopique des modalités d'allaitement et de sevrage à partir des ossements archéologiques
}

\section{Isotopic evidence of breastfeeding and weaning modalities from archaeological bones}

\section{Estelle Herrscher}

Aix Marseille université, CNRS, MCC, LAMPEA UMR 7269, maison méditerranéenne des sciences de l'Homme, 5, rue du Château-de-L'horloge, BP 647, 13094, Aix-en-Provence, France

Reçu le 3 novembre 2012 ; accepté le 7 décembre 2012

Disponible sur Internet le 24 janvier 2013

\section{MOTS CLÉS \\ Allaitement ; Sevrage ; \\ Squelette ; Isotopes stables ; \\ Préhistoire ; \\ Histoire}

\section{KEYWORDS}

Breastfeeding;

Weaning;

Skeleton;

Stable isotopes;

Prehistory;

History

\begin{abstract}
Résumé La plasticité du squelette lui confère la propriété d'enregistrer dans ses tissus certains évènements de sa vie et notamment ceux intervenus durant les premières années de sa vie. Parce que, depuis maintenant plus de 30 ans, le développement instrumental autorise l'analyse du contenu chimique des fossiles avec fiabilité et rapidité, il est possible de cerner chez les populations anciennes les tendances des régimes alimentaires afin d'inférer sur l'histoire de la gestion des ressources alimentaires mais également de détecter la consommation de lait maternel pour reconstituer les pratiques d'allaitement et de sevrage. Après une présentation des marqueurs isotopiques utilisés actuellement pour restituer l'alimentation du jeune enfant, cet article a pour objectif de présenter une synthèse des travaux réalisés et d'en proposer une analyse critique pour souligner leurs avantages et leurs limites.

(c) 2013 Publié par Elsevier Masson SAS pour la Société française de nutrition.
\end{abstract}

Summary The skeletal plasticity confers the property to record different biological events of life, in particular those occurred during the first years. Due to the development of instrumental analysis for over thirty years, the chemical components analysis of fossils allows us to identify dietary trends to understand the history of food resources management as well as the breastfeeding and weaning practices among ancient societies. After a description of chemical markers used today to restore the past dietary practices, this article aims at presenting a synthesis of the published works and discussing them to underline their advantages and limits.

(c) 2013 Published by Elsevier Masson SAS on behalf of Société française de nutrition. 


\section{Introduction}

La femme prend la décision d'allaiter son enfant en fonction de ses capacités physiologiques mais également de sa personnalité, de son éducation, de son entourage et surtout de l'attitude de sa communauté face à ce comportement [1]. Bien plus qu'un processus biologique universel propre aux mammifères, la pratique de l'allaitement au sein est donc à la fois un marqueur socioculturel et biologique des sociétés humaines. Cette pratique s'exprime selon différentes modalités définies par la durée de l'allaitement exclusif et la durée pendant laquelle, en plus de l'allaitement, le jeune enfant voit la diversification de son alimentation; il s'agit de la période sevrage. L' «âge au sevrage», expression couramment utilisée, correspond généralement à l'âge à partir duquel l'enfant est sevré $[2,3]$; il marque ainsi un événement particulier : l'arrêt de consommation du lait maternel. Que ce soit les anthropologues, les historiens et les médecins, tous se sont emparés de ce sujet que ce soit chez les populations passées, traditionnelles et actuelles [4-9]. Chez les populations pré-industrielles, l'allaitement maternel est la seule alternative pour la survie des jeunes enfants. Passé ce premier cap critique, un second les attend: la diversification alimentaire, celle-ci ne doit avoir lieu ni trop tôt, ni trop tard et elle doit être nutritionnellement adaptée et non contaminée [10]. D'un autre côté, un allaitement exclusif long a, d'une part, des implications sur la santé des enfants en raison de son inadéquation face à ses besoins nutritionnels $[11,12]$ et, d'autre part, il peut bloquer l'ovulation et mimer les effets d'un moyen de contraception expliquant les intervalles intergénésiques plus grands observés chez les populations qui pratiquent un allaitement sur une longue durée $[13,14]$.

D'un point de vue anthropologique, l'allaitement et l'âge au sevrage sont donc des données dont les implications sur la mortalité infantile, la fertilité sont capitales pour la compréhension du maintien et de l'évolution des populations d'hier et d'aujourd'hui.

Si la plupart des connaissances acquises aujourd'hui, pour les sociétés pré-industrielles, est l'apanage des historiens, des démographes et des ethnologues [4,15-17], une autre source documentaire, comme les squelettes humains mis au jour dans les cimetières ou sites archéologiques, et plus précisément l'analyse de leur contenu chimique, est maintenant considérée. Depuis une trentaine d'années, l'avancée des techniques liées aux dosages des isotopes stables et des éléments traces et leur application à des contextes archéologiques a permis d'explorer des problématiques relatives à l'alimentation des populations anciennes [18]. Parce que les tissus osseux et dentaires enregistrent des signaux chimiques relatifs à l'environnement dans lequel l'individu a vécu, il est possible de rendre compte de la prédominance de produits marins ou terrestres, de la contribution préférentielle de végétaux ou de viande d'animaux, de la présence de certaines espèces végétales, comme le maiis et le millet dans l'alimentation des populations préhistoriques et historiques [19-24] mais également de rendre compte de la consommation de lait maternel chez les toutpetits [25-27].

Cet article de synthèse a pour objectif de montrer comment les avancées méthodologiques basées sur l'analyse du contenu chimique des ossements et des dents de squelettes archéologiques permettent de caractériser et d'analyser les pratiques d'allaitement des sociétés aujourd'hui disparues, que ce soit à l'échelle individuelle ou populationnelle.

\section{L'allaitement maternel: marqueur biologique et socioculturel}

«Dis moi ce que tu manges, je te dirai qui tu es», cette phrase célèbre de Jean Anthelme Brillat-Savarin a largement trouvé écho dans de nombreux travaux scientifiques étudiant les relations entre la nature des aliments consommés et les pratiques alimentaires d'hier et d'aujourd'hui $[28,29]$. Il en va de même pour l'alimentation des tout-petits pour laquelle le lait maternel, en plus d'être une nourriture à part entière, renvoie à des pratiques très variées que ce soit dans le temps et dans l'espace $[6,12]$. L'allaitement maternel peut être interrompu momentanément dès les premières heures suivant la naissance car, pour certains, le lait produit durant l'expulsion des lochies est considéré comme impropre, ou bien, pour d'autres, le colostrum, de couleur jaunâtre, n'est pas considéré comme mature $[6,30]$. De même, certaines sociétés considérant le lait maternel comme un aliment inadapté à la croissance de l'enfant, administrent en complément soit du lait animal, des bouillies ou des préparations prémâchées dès le plus jeune âge [6]. Puis vient l'apparition des dents déciduales, le retour des menstruations chez la femme ou encore l'arrivée d'un nouvel enfant qui constituent également autant de facteurs modelant les pratiques d'allaitement vers un sevrage progressif (allaitement mixte) ou bien un sevrage brutal [4]. Le lait maternel est également considéré, dans de nombreuses sociétés, comme le vecteur des valeurs physiques mais également morales de la mère et donc empreint d'une forte valeur sacrée et symbolique, résultant de la «cuisson» du sang maternel qui nourrit le fœtus via le cordon ombilical durant la grossesse [30,31]. Enfin, un facteur aussi déterminant dans la prise de décision de la femme d'allaiter est la position du discours religieux et médical. Longtemps associé au discours religieux, les hygiénistes préconisent un allaitement exclusif variant entre six mois et trois ans selon les époques et les lieux mais paradoxalement l'allaitement mercenaire est aussi défendu pour certaines classes sociales dès l'Antiquité jusqu'à l'Époque Moderne $[32,33]$.

Il faut attendre le $x \mathrm{vII}{ }^{\mathrm{e}}$ siècle avec notamment la prise de conscience des ravages causés par l'allaitement mercenaire et l'apparition des biberons non stérilisés sur la survie des jeunes enfants pour que les bienfaits de l'allaitement maternel soient envisagés. Et, ce n'est vraiment, que depuis les années 1970 que le corps médical s'accorde sur les effets positifs de l'allaitement sur la santé des enfants et des mères [34-36] et depuis une vingtaine d'années que cette pratique est recommandée par de nombreuses organisations nationales et internationales $[9,37]$.

Ces quelques données illustrent bien comment, audelà de données purement factuelles comme la durée de l'allaitement et l'âge au sevrage, l'alimentation du jeune enfant est à la fois un marqueur des sociétés et de leurs croyances dans le temps et l'espace et combien les conséquences en termes de survie sont importantes pour comprendre la dynamique des populations et la pérennité de notre espèce.

\section{Des marqueurs chimiques de l'allaitement et du sevrage}

Les tissus osseux et dentaires enregistrent dans leur constitution la nature des aliments à partir desquels ils se synthétisent. L'alimentation du nourrisson se définit : 
- par l'allaitement, c'est-à-dire la consommation d'une nourriture synthétisée par sa mère, conférant à cette alimentation un caractère exclusivement animal ;

- lors du sevrage, par la consommation d'une alimentation supposée plus variée et de nature comparable à celle de l'adulte, intégrant des aliments d'origine végétale (céréales pour la réalisation des galettes ou des bouillies) et des aliments d'origine animale (lait de substitution, viande prémâchée).

L'intérêt est donc de disposer de marqueurs sensibles aussi bien à la nourriture maternelle comme les isotopes stables $^{1}$ de l'azote et de l'oxygène $[25,38,39,41]$ qu'à l'alimentation de sevrage comme les isotopes stables du carbone [38-40] ou les éléments-traces ${ }^{2}$, comme le rapport strontium/calcium [42].

\section{Les marqueurs de la consommation de lait maternel}

Les isotopes stables ${ }^{3}$ de l'azote $\left(\delta^{15} \mathrm{~N}\right.$, rapport $\left.{ }^{15} \mathrm{~N} /{ }^{14} \mathrm{~N}\right)$ sont des marqueurs utilisés depuis de nombreuses années en écologie pour tracer les différents niveaux trophiques d'une chaine alimentaire. Dosées sur la fraction organique des tissus (kératine des cheveux et des ongles, collagène des os et des dents), les signatures isotopiques en azote rendent compte uniquement de la nature protéinique de l'alimentation. Des études réalisées sur des organismes vivants ont montré que les valeurs isotopiques en azote augmentent des niveaux trophiques les plus bas (végétaux) vers les niveaux trophiques les plus élevés (herbivores, puis omnivores et carnivores) [43,44]. À chaque niveau trophique, on observe dans les tissus des organismes un enrichissement en isotope lourd (azote-15), ou fractionnement isotopique, de l'ordre de 3 à $5 \%$, résultant de différents facteurs physiologiques dont le plus important est l'excrétion préférentielle de l'isotope léger (azote-14) $[45,46]$. C'est notamment sur ce principe que se basent les études portant sur l'alimentation du nourrisson, puisque l'enfant allaité entretient avec sa mère une relation de type "proie-prédateur", lui conférant un régime de type «carnivore». Ce principe d'enrichissement en azote-15a notamment été testé à partir d'expériences de nutritions contrôlées sur des couples mères-enfants actuels [35-47].

\footnotetext{
1 Les isotopes stables sont des marqueurs fréquemment utilisés en archéologie car, lorsque la conservation le permet, leurs teneurs restent inchangées dans les tissus après la mort de l'individu. Les isotopes d'un élément ont des masses différentes (nombre de neutrons) et un nombre identique d'électrons. Ainsi, les deux isotopes d'un même élément (lourd: ${ }^{15} \mathrm{~N},{ }^{13} \mathrm{C} /$ léger: ${ }^{14} \mathrm{~N},{ }^{12} \mathrm{C}$ ) réagissent dans les réactions chimiques avec une cinétique plus rapide pour l'isotope léger.

2 Les éléments traces sont le strontium, cuivre, fer, zinc, baryum, calcium. Leur teneur exprimée en ppm est corrélée à la nature des aliments consommés. Par exemple, les crustacés, la viande sont riches en cuivre [48].

3 Les résultats des mesures des compositions isotopiques sont notés $\delta$ et exprimés en $\%$ a afin de visualiser les variations très faibles entre les isotopes d'un même élément. Les compositions isotopiques correspondent au rapport de l'isotope lourd sur l'isotope léger dans un échantillon comparé à ce même rapport dans un standard. Par exemple, pour le carbone: $\delta^{13} \mathrm{C}=\left[\left[\left({ }^{13} \mathrm{C} /{ }^{12} \mathrm{C}\right.\right.\right.$ échantillon $) /\left({ }^{13} \mathrm{C} /{ }^{12} \mathrm{C}\right.$ standard $\left.\left.)\right]-1\right] \times 1000 \%$
}

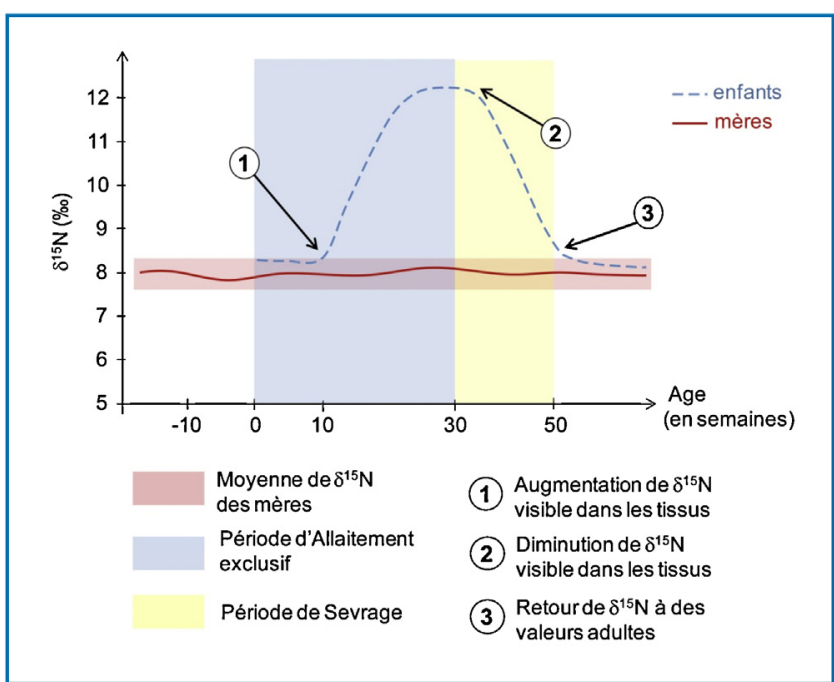

Figure 1. Évolution des compositions isotopiques en azote $\left(\delta^{15} \mathrm{~N}\right)$ dans les tissus de couples mères-enfants durant la période d'allaitement et de sevrage. D'après $[25,47]$.

À partir de l'analyse longitudinale 4 d'un couple «mèreenfant» et de l'analyse transversale de 16 couples «mèreenfant», Fogel et al., en 1989 [25], mettent en évidence trois faits importants:

- les tissus des nouveau-nés à la naissance ont une signature isotopique en azote similaire à celle de leur mère, car les tissus du nouveau-né sont synthétisés in utero à partir des nutriments disponibles via l'alimentation de sa mère;

- à partir de trois mois, les tissus des enfants allaités s'enrichissement progressivement en azote15 correspondant au temps nécessaire pour «épuiser» le pool nutritionnel venant de la mère et commencer à synthétiser des tissus sur les nouveaux nutriments apportés par le lait maternel ;

- lorsque l'enfant est sevré, les tissus des enfants présentent progressivement un appauvrissement en azote15 témoignant de la diminution de la consommation de produits lactés maternels.

En 2006, Fuller et al. poursuivent cette étude en augmentant le corpus, avec le suivi longitudinal ${ }^{5}$ de six couples «mère-enfant » avec quatre couples d'enfants allaités exclusivement et deux couples d'enfants nourris avec des laits de substitution, animal ou végétal. Ces travaux ont permis :

- de quantifier plus rigoureusement cet enrichissement en azote-15 entre 2 et $4 \%$ lors de la période d'allaitement ;

- de confirmer l'apparition de cet enrichissement dès la dixième semaine ;

- de démontrer que les rapports isotopiques en azote tracent la durée de la consommation de lait maternel.

Ainsi, l'évolution des signatures isotopiques en azote attendue dans des tissus d'enfants au cours du processus d'allaitement et de sevrage décrit une courbe en cloche, dont le sommet marquerait le début de la diminution d'une alimentation exclusivement maternelle (Fig. 1). La

\footnotetext{
${ }^{4}$ Les dosages isotopiques ont été réalisés sur les ongles des mères et des enfants.

5 Les dosages isotopiques ont été réalisés sur les cheveux ou les ongles des mères et des enfants.
} 


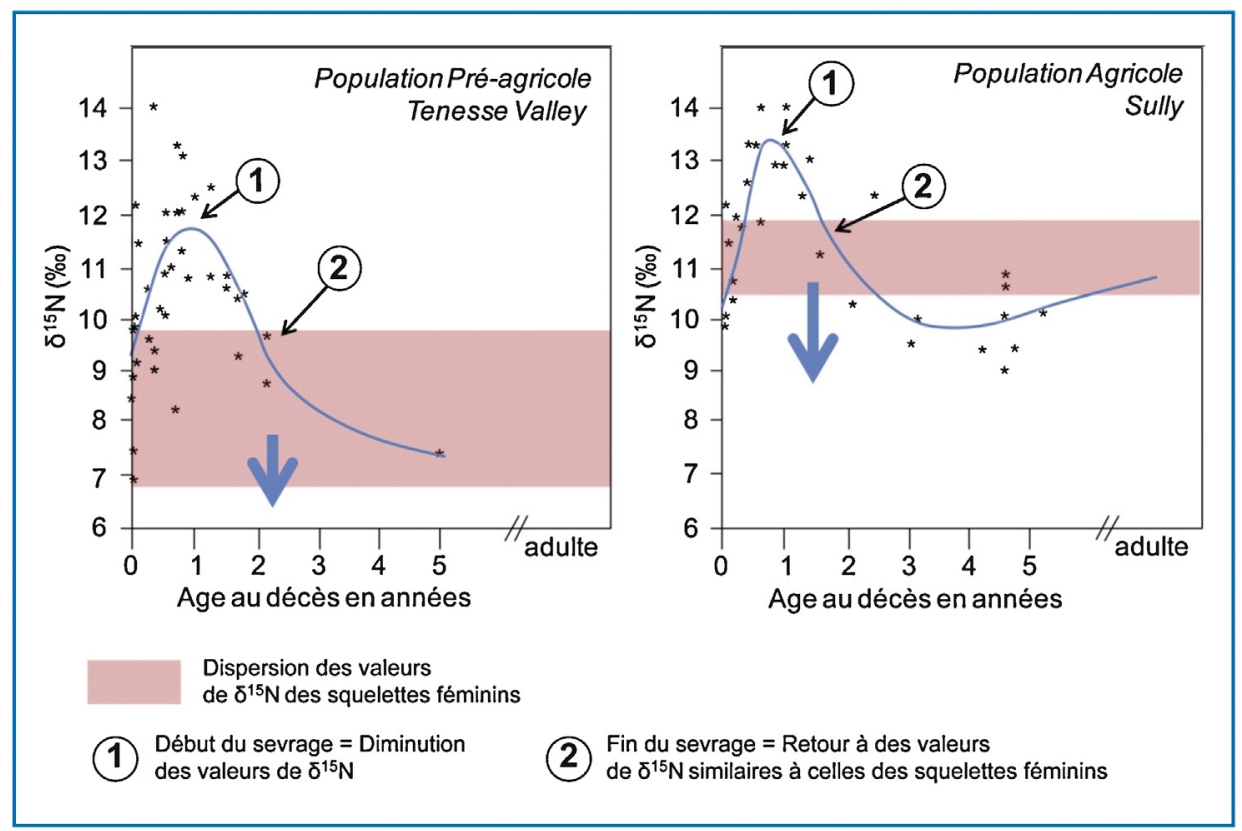

Figure 2. Évolution des compositions isotopiques en azote $\left(\delta^{15} \mathrm{~N}\right)$ en fonction de l'âge au décès de sujets immatures issues de sociétés préagricoles et agricoles en Amérique du nord. D'après [25].

majorité des travaux réalisés sur les squelettes découverts en contexte archéologique repose sur ces considérations et s'attache donc à traquer la diminution des valeurs de $\delta^{15} \mathrm{~N}$ en fonction de l'âge au décès des enfants au sein de la cohorte pour estimer la durée de la consommation de lait maternel. Le transfert de ce modèle à l'étude d'une population archéologique nécessite toutefois quelque prudence. Le processus dynamique de l'allaitement et du sevrage est décrit de façon transversale, avec un seul dosage par individu qui, dans le cas d'un prélèvement osseux, correspond à l'alimentation des derniers moments de sa vie, soit quelques semaines pour les sujets les plus jeunes ou quelques mois pour les plus âgés. Les mères ne sont pas analysées en tant qu'individus appariés aux enfants. L'enrichissement en azote-15 dans les tissus des enfants est donc apprécié par comparaison avec la variabilité isotopique féminine propre à chaque population archéologique afin de tenir compte de ses propres caractéristiques alimentaires ${ }^{6}$. Enfin, le temps de latence nécessaire pour l'enregistrement d'un nouveau régime alimentaire dans les tissus osseux ou dentaires n'est pas connu avec certitude, il est estimé à quelques mois ${ }^{7}$.

Les premières applications ont été réalisées sur des populations archéologiques nord-américaines natives précontact et postcontact européen. Les premiers résultats ont ainsi montré un sevrage plus précoce pour la population agricole de Sully (1650-1700 après J.-C.) comparativement à la population native pré-horticole de Tennessee Valley (5500-200 avant J.-C.) [25]. Ces résultats s'accordaient

\footnotetext{
${ }^{6}$ Les échantillons archéologiques sont issus de cimetières, la mortalité des enfants n'étant pas celles des mères, il est utopique de pouvoir constituer un échantillon de couples «mère-enfant».

7 Les seuls travaux réalisés sur du collagène osseux en contexte de nutrition contrôlée concerne des jeunes bovidés, d'autres données sont disponibles sur les cheveux, associés aux données médicales sur le taux de renouvellement des protéines tissulaires et les études sur les cheveux et les ongles de nouveaux nés, on peut penser que 6 mois sont nécessaires pour détecter une modification alimentaire dans des tissus osseux de squelettes immatures [53].
}

parfaitement avec l'hypothèse selon laquelle la diminution de l'âge au sevrage serait à l'origine d'une plus forte fertilité des femmes et consécutivement d'une croissance plus importante des populations [49] (Fig. 2). Toutefois, dans un article de synthèse basé sur un corpus plus important de populations préagricoles (Carlston Annis, Indian Knoll, 3500-1500 avant J.-C.) et agricoles (Angel, Tinsley Hill, 1300-1450 après J.-C.) intégrant des données isotopiques et paléodémographiques, Schurr et al. montrent plus tard que l'âge au sevrage est relativement uniforme entre toutes les populations, qu'elles soient pré-agricoles ou agricoles. Ainsi, la diminution de l'âge au sevrage ne serait finalement pas en rapport avec le développement de l'agriculture mais plutôt à mettre en relation avec la sédentarisation des groupes humains [50,51]. A contrario, une étude comparative entre deux populations natives agricoles (de Angel (1300-1450 après J.-C.) et de Mac Pherson (contact européen, $\mathrm{xvI}^{\mathrm{e}}$ siècle après J.-C.) et une population d'immigrés européens installés au Canada (Prospect Hill, 1824-1879 après J.-C.) montre, que c'est au moment du contact que l'âge au sevrage est le plus précoce et la fertilité la plus importante, probablement en réponse au stress lié à la colonisation [26,52]. La variabilité importante des pratiques d'allaitement observées démontrent la complexité des réponses culturelles adoptées par les groupes humains pour assurer leur pérennité [27]. La démocratisation des outils isotopiques et le nombre croissant d'opérations archéologiques ont depuis permis le développement de ce type de recherches dans d'autres régions du monde et pour d'autres périodes. Par exemple, ces pratiques analysées chez deux populations néolithiques contemporaines découvertes en Turquie (Asikli Höyük et Cayönü Tepesi, $\mathrm{x}^{\mathrm{e}}-\mathrm{xV} \|^{\mathrm{e}}$ millénaires avant J.-C.) ont révélé une diminution de la consommation de lait maternel plus précoce, vers un an, pour l'une et plus tardif, vers deux ans, pour l'autre, associée à une mortalité infantile des enfants de moins de cinq ans plus importante chez la population pratiquant l'allaitement le plus long [54]. Un climat plus clément et une gestion différente des ressources végétales 
et animales expliqueraient le succès d'un sevrage plus précoce [54]. La période historique la plus documentée à partir des analyses isotopiques est l'Antiquité tardive ( $\mathrm{I}^{\mathrm{er}}$ - $\mathrm{V}$ e siècles après J.-C.), qu'il s'agisse de populations ayant vécu en Angleterre, en Italie et même sous domination romaine en Égypte. Ces recherches ont démontré une diminution de la consommation de lait maternel dès six à huit mois et son arrêt complet vers deux à trois ans en accord avec les recommandations des hygiénistes du monde romain, comme Soranus et Galien, ainsi que la persistance de ces pratiques au-delà de la période sous domination romaine (Fig. 3) $[39,55,56]$. Couvrant une large période $d u x^{e}$ au $x v l^{e}$ siècle après J.-C., les études conduites sur la population médiévale anglaise de Wharram Percy ont révélé une pratique uniforme avec une cessation de la nourriture maternelle entre un et deux ans corroborant également les discours hygiénistes de l'époque [57-59].

Les isotopes stables de l'oxygène $\left(\delta^{18} \mathrm{O},{ }^{18} \mathrm{O} /{ }^{16} \mathrm{O}\right)$, dosés dans la fraction minérale des ossements, ont également été utilisés pour marquer la consommation de lait maternel $[40,41]$. Les valeurs de $\delta^{18} \mathrm{O}$ des organismes sont liées à celles de l'eau météorique, ingérée par l'eau de boisson et l'eau contenue dans les aliments. À ce titre, l'oxygène est plus un marqueur des caractéristiques environnementales, comme le climat et l'altitude. Toutefois certains auteurs ont également mesuré des valeurs de $\delta^{18} \mathrm{O}$ plus élevées, comprises entre 0,5 et $2 \%$, dans des tissus synthétisés durant la période d'allaitement chez l'animal et l'homme [40]. Le lait est enrichi en oxygène-18 par rapport à l'eau ingérée par la mère, une diminution des valeurs de $\delta^{18} O$ dans les tissus peut donc s'interpréter comme un arrêt de la consommation de lait maternel. L'analyse de ce marqueur sur l'émail dentaire de molaires de 35 individus découverts dans le site de Kaminaljuyù au Guatemala (700 avant J.-C. -1500 après J.-C.) a permis de mettre en évidence un allaitement long au-delà de quatre ans ainsi qu'un sevrage plus tardif [40]. En revanche, Williams et collaborateurs ne détectent pas de modifications des valeurs de $\delta^{18} 0$ dans les ossements de

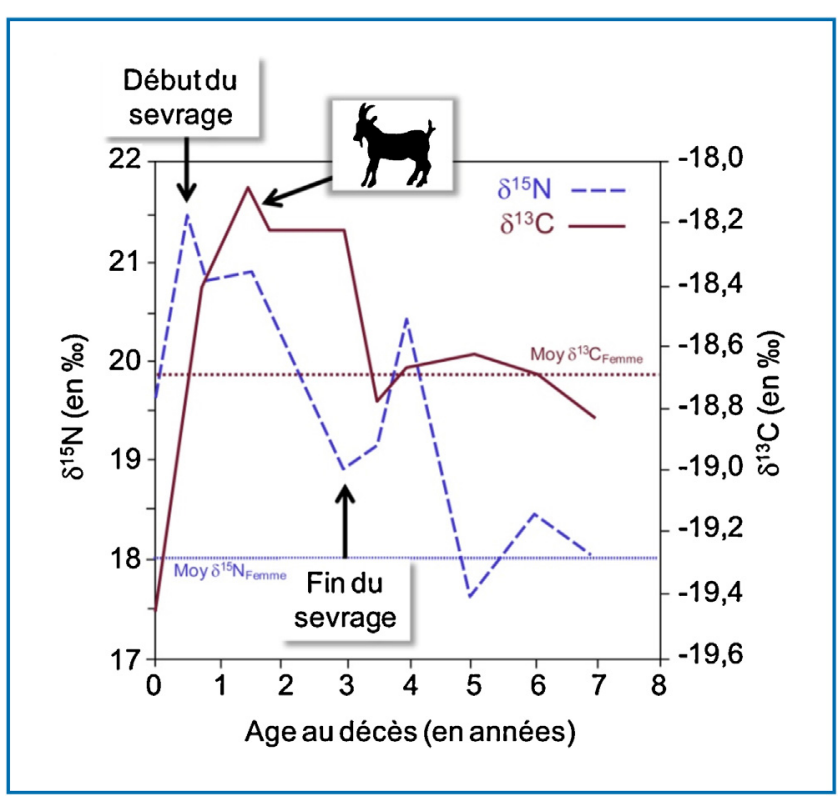

Figure 3. Distribution des compositions isotopiques en carbone et en azote en fonction de l'âge au décès chez des enfants mis au jour dans la nécropole de Kellis 2, dans l'oasis de Dakhleh en Égypte (250-450 après J.-C.) D'après [39]. jeunes enfants Maya alors que les valeurs de $\delta^{15} \mathrm{~N}$ révèlent l'arrêt de la consommation de lait maternel [60]. Cette différence pourrait éventuellement s'expliquer par la nature de la fraction analysée, les premiers auteurs ont dosé le $\delta^{18} \mathrm{O}$ sur les phosphates de l'émail dentaire alors que les seconds l'ont dosé sur les carbonates du tissu osseux. L'usage de ce marqueur pour détecter le sevrage reste peu fréquent dans les études archéologiques.

\section{Les marqueurs de la nourriture de sevrage}

Des marqueurs peuvent également être utilisés pour suivre, cette fois, l'introduction de l'alimentation de sevrage, qui peut être soit un lait animal, soit des bouillies ou galettes. Les isotopes stables du carbone de la fraction organique permettent de discriminer la nature des protéines végétales selon qu'il s'agit d'espèces végétales utilisant une photosynthèse en $C_{3}$, comme la plupart des végétaux d'environnement tempéré comme par exemple le blé, l'avoine et l'orge (valeurs basses de $\delta^{13} \mathrm{C}$ ), ou d'espèces végétales utilisant une photosynthèse en $\mathrm{C}_{4}$ comme certaines graminées, le maïs, le millet ou le sorgho (valeurs hautes de $\delta^{13} \mathrm{C}$ ) [61-63]. Les caractéristiques isotopiques de plantes s'enregistrent ensuite dans les tissus des consommateurs herbivores, puis dans ceux des carnivores.

Ainsi dans certaines régions du monde, une augmentation des valeurs des compositions isotopiques en carbone dans les tissus des jeunes enfants peut témoigner d'une nourriture de sevrage spécifique, élaborée à partir de maïs ou de millet ou la consommation du lait d'un animal ayant lui-même consommé des céréales particulières. Dans la population nord-américaine native de MacPherson, datée $\mathrm{du} X \mathrm{VVI}^{\mathrm{e}}$ siècle après J.-C., l'augmentation des valeurs de $\delta^{13} \mathrm{C}$ dans les tissus de jeunes enfants en cours de sevrage a permis d'identifier la consommation de bouillies ou galettes cuisinées à partir de maïs [38]. De façon similaire, l'analyse couplée des isotopes de l'azote et du carbone chez des enfants mis au jour dans la nécropole de Kellis 2, dans l'oasis de Dakhleh en Egypte, alors sous domination romaine (250-450 après J.-C.) a révélé un enrichissement en carbone-13, vers six mois, suivi d'un retour progressif des valeurs de $\delta^{13} \mathrm{C}$ vers des valeurs adultes, à l'âge de deux à trois ans (Fig. 3) [39]. L'analyse isotopique des collagènes animaux du cheptel de cette population a révélé des valeurs de $\delta^{13} \mathrm{C}$ plus élevées que celles des sujets humains, indiquant la consommation de graminées en $\mathrm{C}_{4}$ et de millet par les animaux. Cette observation a ainsi permis aux auteurs de confirmer l'hypothèse d'une nourriture de sevrage constitué de lait de chèvre [39].

Les isotopes stables du carbone dosés dans la fraction minérale des ossements permettent non seulement de caractériser la nature protéinique mais également la nature lipidique et glucidique des aliments. Les lipides étant appauvris en carbone-13 par rapport aux autres macronutriments [64], l'introduction d'une alimentation de sevrage, par définition moins riche en lipide que le lait maternel, conduit à des valeurs de $\delta^{13} \mathrm{C}$ plus élevées dans les tissus des enfants en période de sevrage. Cette augmentation des valeurs de $\delta^{13} \mathrm{C}$ est d'autant plus marquée que la nourriture de sevrage est pauvre en lipide [40].

Enfin, un dernier marqueur mérite d'être mentionné, il s'agit du rapport strontium/calcium. D'un niveau trophique à l'autre, dans les tissus de mammifères, le strontium est préférentiellement bio-épuré face au calcium, ainsi plus l'organisme se trouve en haut de la chaine alimentaire, et plus les valeurs du rapport $\mathrm{Sr} / \mathrm{Ca}$ diminuent [65]. Le 
lait présente donc des valeurs du rapport $\mathrm{Sr} / \mathrm{Ca}$ nettement plus basses que les végétaux. Ce principe a permis à Sillen et Smith [42], dans une étude publiée en 1984, de présenter les premiers résultats concernant l'alimentation de jeunes enfants découverts sur le site de Dor en Israël, daté entre 800 et 1300 après J.-C.. Les résultats ont montré que les enfants âgés entre deux et trois ans étaient tous sevrés. Toutefois, en raison de la mise en évidence d'une discrimination différentielle du strontium par le système gastro-instestinal au cours de la croissance, l'usage de ce marqueur pour la reconstitution des pratiques alimentaires n'a jusqu'à aujourd'hui jamais été reconduit.

\section{Détection des modalités de pratiques d'allaitement à l'échelle de l'individu}

Au-delà des différents marqueurs permettant de caractériser l'alimentation maternelle et celle de sevrage, plusieurs approches peuvent être développées impliquant, d'une part, soit le tissu osseux soit le tissu dentaire et, d'autre part, soit une analyse par individu ou deux ou plusieurs analyses par individu. Dans le cas d'une analyse interindividuelle des signatures chimiques dans une population de sujets immatures, l'objectif est de cerner à partir de quel âge les valeurs des enfants rejoignent celles des valeurs des adultes, signifiant l'arrêt de l'allaitement et/ou l'introduction du régime de sevrage. Toutefois dans certains cas, cette approche se révèle difficile à mettre en œuvre pour deux raisons principales:

- la variabilité isotopique adulte peut être tellement importante qu'elle peut masquer celle des enfants induites par l'allaitement/sevrage;

- l'indisponibilité des squelettes féminins pour des raisons de conservation peut empêcher de définir cette variabilité adulte.

C'est notamment pour s'affranchir de la variabilité adulte tout en apportant des réponses à l'échelle individuelle, que certains auteurs proposent une approche basée sur l'analyse intra-individuelle des marqueurs. Une telle approche repose sur la mise en place d'une stratégie d'échantillonnage de plusieurs tissus au sein d'un squelette pour détecter un changement de l'alimentation au cours de la vie de l'individu. Dans le cas qui nous intéresse, deux modifications alimentaires interviennent: le passage de l'alimentation de la mère héritée et enregistrée dans les tissus de l'enfant à la naissance à l'allaitement, puis le passage de l'allaitement à la nourriture de sevrage $8[40,66,67]$. L'origine des signaux alimentaires enregistrés au niveau du squelette humain diffère selon leur nature. Le tissu osseux subissant un remodelage permanent de sa trame organique et minérale, le signal enregistré correspond à la moyenne des alimentations précédant le décès du sujet alors que le tissu dentaire, qu'il s'agisse de l'émail ou la dentine, une fois en place, ne subit plus de modification de sa constitution, le signal enregistré correspond alors à l'alimentation la plus contemporaine du moment de sa formation. Parce que la croissance des dents répond à un calendrier de minéralisation et d'éruption dentaire bien établi et presque universel

\footnotetext{
8 Depuis les années 1990 , cette approche est également utilisée pour documenter les modifications alimentaires au cours de la vie dans des contextes de migrations de populations, de colonisation ou encore la saisonnalité d'un événement comme la mort $[67,72]$.
}

il est possible à partir de deux échantillons dentaires, par exemple la couronne d'une première molaire permanente et celle d'une troisième molaire d'inférer sur des évènements alimentaires survenus respectivement entre neuf mois et trois ans et entre dix et 12 ans [68]. En outre, cette approche permet de considérer non plus des sujets immatures décédés, c'est-à-dire les non-survivants, mais des sujets adultes ayant survécu à la période de sevrage. Une comparaison de cette approche appliquée sur des sujets immatures et adultes d'une même population médiévale anglaise (Wharram Percy, $x^{\mathrm{e}}-\mathrm{xv}^{\mathrm{e}}$ siècles après J.-C.) n'a toutefois montré aucune divergence des patrons d'allaitement entre nonsurvivants et survivants confortant ainsi l'analyse des tissus des jeunes enfants pour ces problématiques alimentaires [59].

Un même individu peut également enregistrer et conserver, dans ses tissus osseux et dentaires des signaux relatifs à différents moments de sa vie. Cette stratégie d'échantillonnage sur tissu osseux versus tissu dentaire a été validée par une étude des compositions isotopiques en azote en contexte de nutrition contrôlée réalisée sur cinq jeunes taurillons sevrés au moment de leur abattage [67]. Ces travaux ont ainsi démontré :

- qu'au niveau de la racine d'une dent en cours de minéralisation ${ }^{9}$, le signal isotopique en azote enregistré est celui de son alimentation de sevrage au moment de l'abattage (valeur basse de $\delta^{15} \mathrm{~N}$ );

- que le tissu osseux conserve lui des traces de l'alimentation lactée maternelle, présente six mois avant l'abattage (valeur haute de $\delta^{15} \mathrm{~N}$ ) car le collagène osseux a enregistré progressivement l'alimentation maternelle en remplacement du signal isotopique hérité de sa mère à la naissance [67].

Cette stratégie d'échantillonnage reposant sur la différence des valeurs isotopiques entre les deux tissus permet ainsi d'identifier, pour chaque individu, son statut par rapport au sevrage (allaité/sevré) (Fig. 4). Cette approche a été appliquée à trois populations couvrant la période du Moyen Âge et de l'Époque moderne en France ( $\mathrm{VIII}^{\mathrm{e}}-\mathrm{xVIII}{ }^{\mathrm{e}}$ siècles) $[53,69,70]$. À partir de l'analyse de 51 sujets immatures, d'âge au décès compris entre six mois et cinq ans, les résultats isotopiques attestent d'une mosaïque de comportements alimentaires adoptés envers les enfants qui ne seraient en relation ni avec l'âge au décès des enfants, ni avec la période chronologique considérée. Alors que pour le bas Moyen âge $\left(\mathrm{xIII}^{\mathrm{e}}-\mathrm{xV}^{\mathrm{e}}\right.$ siècles), une seule pratique se distingue avec un sevrage tardif vers 2,5 ans [53,71], pour les périodes carolingiennes $\left(\mathrm{VIII}^{\mathrm{e}}-\mathrm{IX}^{\mathrm{e}}\right.$ siècles) et modernes $\left(\mathrm{XVII}^{\mathrm{e}}-\mathrm{xVIII}{ }^{\mathrm{e}}\right.$ siècles), deux pratiques ont été mises en évidence $[69,70]$. Ces travaux ont permis de montrer que pour une même période, certains enfants pouvaient être sevrés vers six mois à un an alors que d'autres enfants étaient encore allaités au-delà de trois ans $[69,70]$. Si une telle pratique double pourrait s'expliquer par le recours plus fréquent à des nourrices à l'Époque moderne, d'autres facteurs doivent être évoqués pour la période carolingienne (Fig. 5). Que ce soit le discours religieux ou les recommandations des hygiénistes, les données historiques restent éparses pour cette période [73] ; elles s'accordent pour recommander un sevrage entre deux et trois ans. Au-delà du fait que

\footnotetext{
${ }^{9}$ Les racines en cours de minéralisation présentent des apex non fusionnés dont la croissance se poursuivait au moment de la mort du sujet.
} 


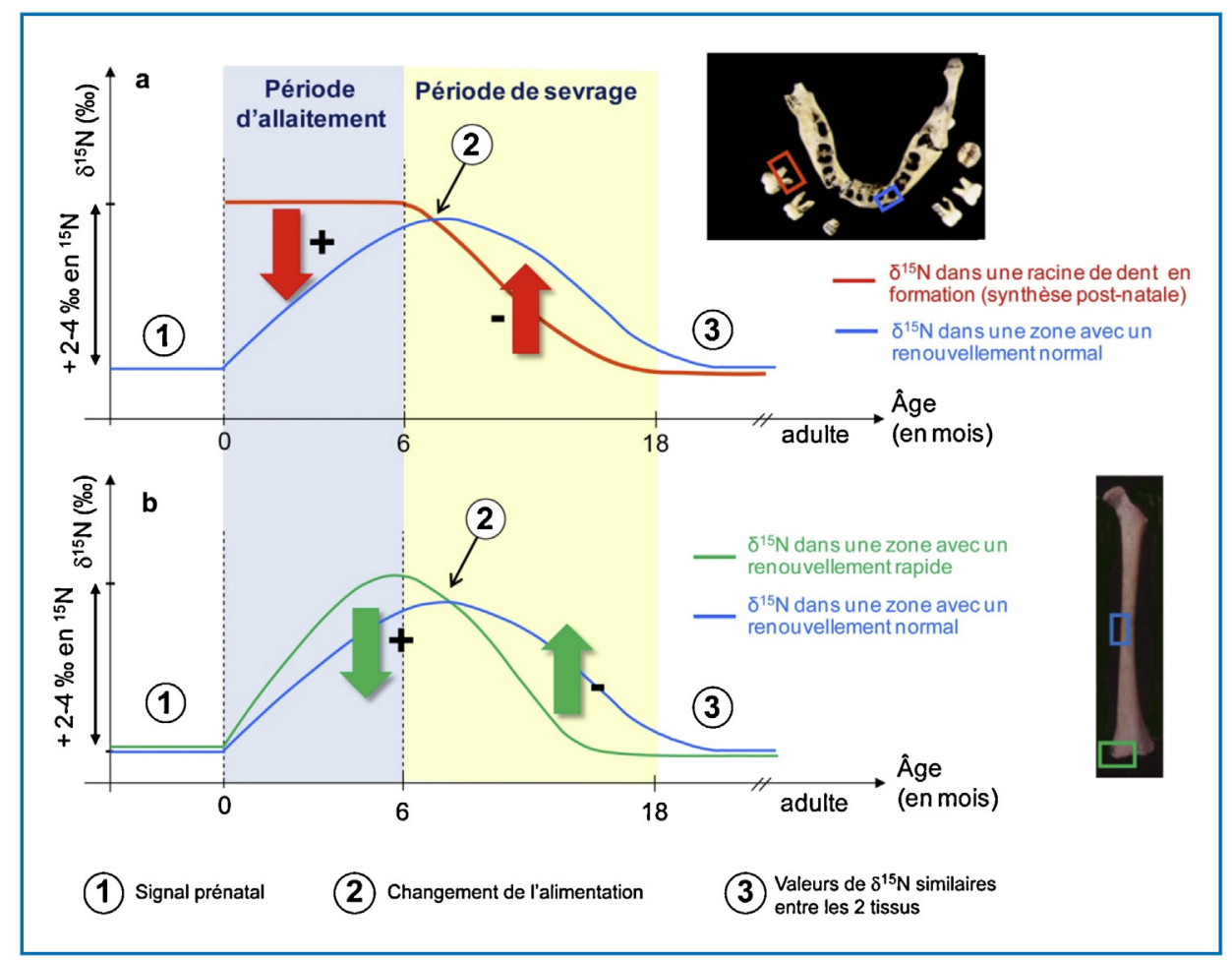

Figure 4. Analyse intra-individuelle : évolution des compositions isotopiques en azote $\left(\delta^{15} \mathrm{~N}\right)$ durant la période d'allaitement et du sevrage pour deux tissus prélevés: a: prélèvements aux niveaux d'une racine de dent en formation et d'un fragment osseux dans une zone en renouvellement normal ; $b$ : prélèvements de deux tissus osseux: l'un au niveau d'une zone en renouvellement rapide et l'autre au niveau d'une zone en renouvellement normal. Modifié d'après [53].

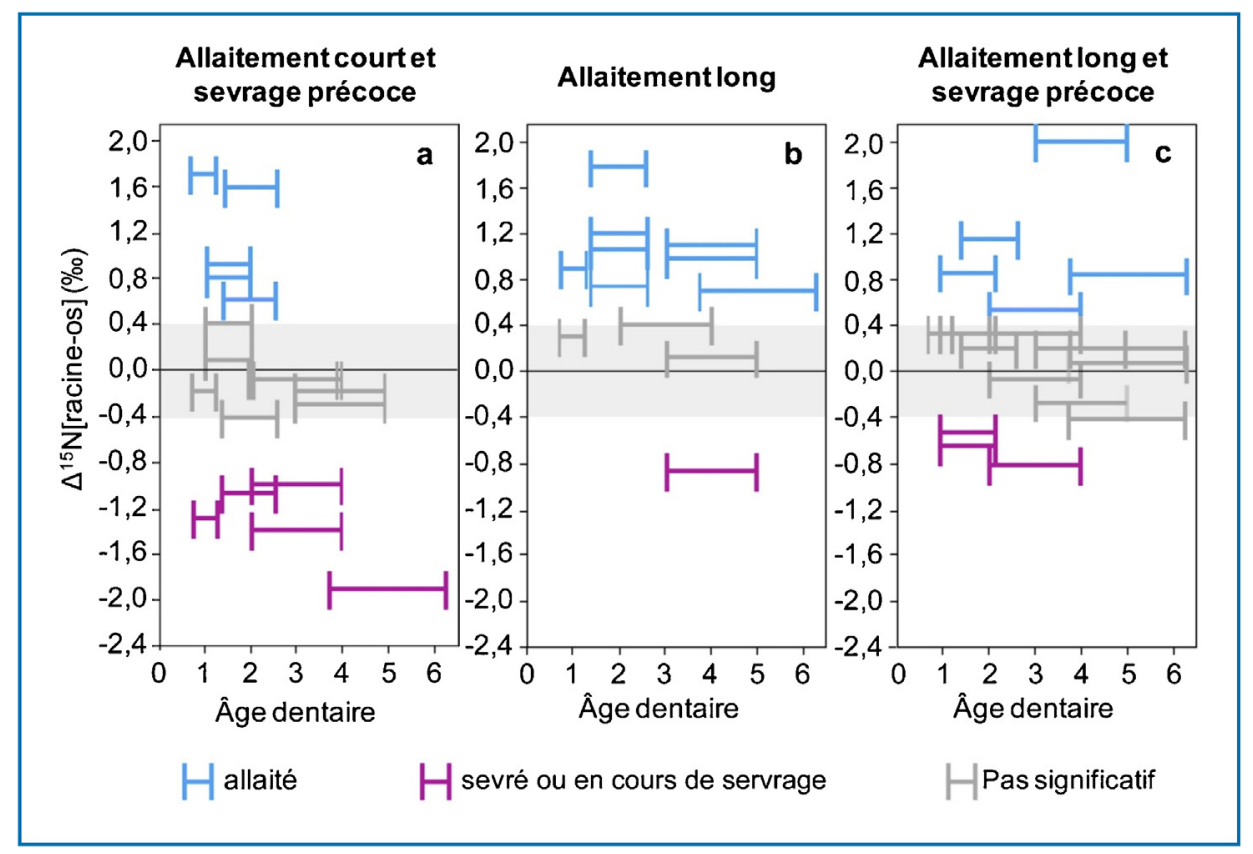

Figure 5. Différence de valeurs de $\delta^{15} \mathrm{~N}$ entre les deux tissus prélevés (racine en formation et os) : a: période mérovingienne, (vIII $-1 \mathrm{X}^{\mathrm{e}}$ s., Saint-Martin-des-Champs, [70]); b : période de la fin du Moyen Âge ( $\mathrm{xIII}{ }^{\mathrm{e}}-\mathrm{xV}{ }^{\mathrm{e}}$ s., Saint-Laurent, [53]); c: époque moderne (xvII $-\mathrm{xvIII}{ }^{\mathrm{e}}$ s., Saint-Laurent, [69]). Les «âges dentaires» correspondent à des intervalles d'âges au décès estimés en années à partir du degré de minéralisation des dents déciduales et permanentes [68].

ces travaux montrent que les recommandations n'étaient pas toujours suivies, ils soulignent le fait que si l'on veut comprendre les modalités d'allaitement et de sevrage dans le passé, et notamment apprécier la diversité au niveau de chaque population, il est nécessaire de développer des stratégies d'échantillonnage intra-individuel.
Lors de travaux pionniers menés sur des ossements animaux archéologiques, Fizet (1992) montre qu'au niveau d'un même os, sur un même individu, des valeurs différentes de $\delta^{15} \mathrm{~N}$ peuvent s'enregistrer selon la zone de prélèvement analysée [74] (Fig. 4). Ces différences résultent de la persistance d'un régime alimentaire antérieur au sevrage, 


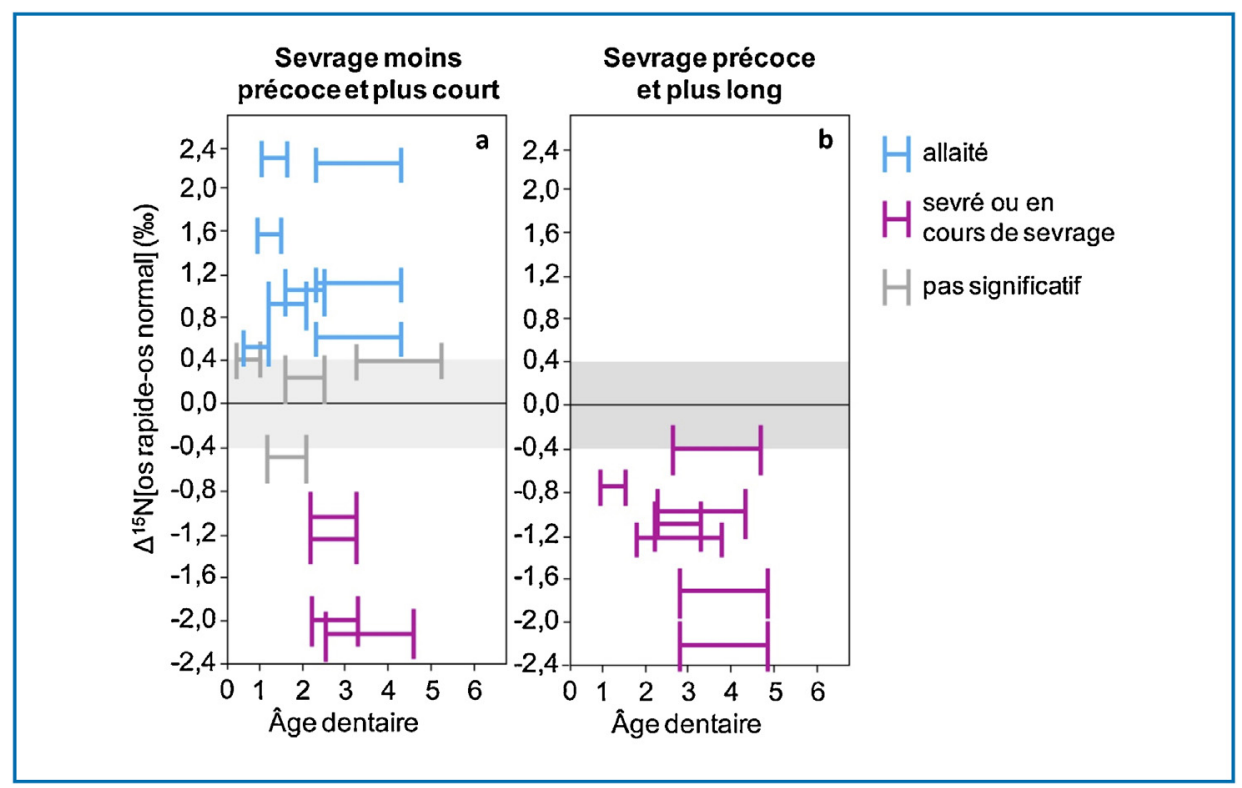

Figure 6. Différence de valeurs de $\delta^{15} \mathrm{~N}$ entre les deux tissus prélevés (os à renouvellement rapide/normal) : $\mathrm{a}$ : néolithique ancien; $\mathrm{b}$ : néolithique plus récent (Sibérie, 6850-3250 avant J.-C.) [75]. Les «âges dentaires» correspondent à des intervalles d'âges au décès estimés en années à partir du degré de minéralisation des dents déciduales et permanentes [68].

c'est-à-dire le lait maternel dans la partie de l'os où le renouvellement est le plus lent. Suivant une approche similaire, Waters-Rist et al. ont développé une stratégie d'échantillonnage spécifique sur des os longs d'enfants, en prélevant un fragment à proximité d'une surface métaphysaire, où le renouvellement est rapide, et un fragment au niveau de la corticale de la diaphyse, où le renouvellement est lent [75]. Cette stratégie appliquée à deux populations néolithiques ayant vécu en Sibérie (6850-3250 avant J.-C., 23 enfants âgés entre zéro et trois ans) a permis de montrer qu'au cours de cette période si un allaitement mixte pouvait durer jusqu'à trois à quatre ans, alors les modalités de sevrage étaient différentes entre le groupe néolithique le plus ancien et le plus récent (Fig. 6). Les auteurs ont ainsi mis en évidence que la nourriture de sevrage était introduite plus précocement et la période de l'allaitement mixte s'allongeait au cours du Néolithique.

\section{Discussion et conclusion}

À raison d'un dosage ou de plusieurs dosages par individu et de la disponibilité d'une cohorte numériquement bien représentée, nous avons montré qu'il est possible, chez les populations archéologiques, d'estimer:

- la durée de la consommation de lait maternel et l'âge d'introduction d'une nourriture de sevrage;

- le statut de chaque enfant par rapport au sevrage dans le but d'apprécier l'homogénéité de ces pratiques.

D'un point de vue général, les modalités de pratiques d'allaitement/sevrage observées répondent à des déterminants biologiques universels avec une diminution de la consommation de lait maternel compris entre six mois et deux ans et un arrêt de celui-ci compris entre trois et quatre ans. En cela, ces travaux corroborent les études portant sur les primates ou les sociétés traditionnelles et s'accordent sur:

- un allaitement physiologique naturel possible chez l'homme [76];
- une variabilité interspécifique de la durée de l'allaitement plus importante que celle existante à l'intérieur d'une même espèce.

L'analyse isotopique d'enfants de populations natives nord-américaines précontact européen a révélé qu'un âge au sevrage précoce n'était pas systématiquement en relation avec un accroissement de la population [50]. Si ce résultat est consistent avec un mode de vie sédentaire, en revanche une étude réalisée sur une population de chasseurs-cueilleurs d'Afrique du Sud confirme un allaitement long associé également à un sevrage long jusqu'à l'éruption complète de la denture déciduale [77]. Des stratégies de pluri-échantillonnages de tissus dentaires et/ou osseux ont, quant à elles, permis de montrer que les modalités de mises en place du sevrage étaient indépendantes de la période préhistorique ou historique considérée. En effet, que ce soit au Néolithique chez des populations de Sibérie, à l'Époque mérovingienne ou à l'Époque moderne chez des populations urbaines françaises, les femmes pouvaient choisir d'adopter un allaitement exclusif et une durée de sevrage plus ou moins longue $[69,70,75]$ ). Ainsi, sans remettre en cause les résultats obtenus sur des populations antiques, attestant du suivi des recommandations des hygiénistes contemporains, ces recherches démontrent que le choix des femmes d'allaiter leurs enfants ne suit pas toujours les règles imposées et que des facteurs comme la disponibilité des ressources alimentaires, qu'elles soient végétales ou animales, le contexte social et sanitaire sont autant de déterminants à considérer si l'on veut mieux comprendre les pratiques d'allaitement dans le passé.

En regard de certains travaux d'historiens ou d'ethnologues, les résultats fournis par l'étude du contenu chimique des ossements peuvent apparaître imprécis, comme par exemple entre six mois et un an pour le début du sevrage et un arrêt vers trois à quatre ans de l'allaitement. Ces imprécisions inhérentes à la nature même de nos corpus d'étude sont de plusieurs ordres. Premièrement, elles résultent d'un biais taphonomique, qui en altérant la conservation quantitative et qualitative des ossements, conduit à des études basées sur des 
corpus numériquement faibles en regard de l'attribution chronologique de certains sites. Deuxièmement, elles sont consécutives à des biais méthodologiques comme :

- l'imprécision de l'estimation de l'âge au décès d'un squelette ;

- l'absence de données fiables sur le temps nécessaire pour que les tissus osseux ou dentaires enregistrent dans leur composition la nouvelle alimentation.

Malgré ces limites, l'analyse directe de l'alimentation enregistrée dans les tissus biologiques fossiles, permet de $s$ 'affranchir de l'étude des lésions osseuses ou dentaires ${ }^{10}$ dont l'expression consécutive au stress du sevrage n'est pas obligatoire chez tous les individus. Elle permet également de s'affranchir du biais social des données historiques. En effet, alors que les données historiques sont généralement issues de récits relatant la vie de certains groupes sociaux, notamment celle des bourgeois ou des dirigeants, l'étude des ossements archéologiques s'intéressent à l'ensemble des individus de populations pouvant être socialement plus diversifiées. Le développement croissant de nouveaux outils biogéochimiques dédiées aux problématiques anthropologiques $[78,79]$, les avancées méthodologiques en termes d'équipements de mesures, plus fiables (micro-échantillonnage des tissus), plus rapides (analyses pluri-éléments), ainsi que la découverte de nouveaux sites archéologiques devraient permettre dans un futur proche de poursuivre une exploration plus fiable des pratiques d'allaitement des sociétés disparues.

Sevrage précoce ou sevrage tardif, telle est la question? En effet, les sevrages avant l'âge de un an sont peu fréquents et sont essentiellement le fait de l'expérience occidentale actuelle. Les sociétés industrialisées ont vu un net recul de l'allaitement au sein pour plusieurs raisons, l'apparition de laits maternisés et de biberons de mieux en mieux adaptés aux besoins de l'enfant, l'activité professionnelle des femmes mais également le souci de ces dernières de ne pas altérer leur corps. Malgré un discours médical et une opinion publique nettement en faveur de l'allaitement au sein [80], aujourd'hui seules $30 \%$ des femmes françaises allaitent encore à un mois contre $60 \%$ d'allaitement à la naissance pour les femmes américaines et $98 \%$ pendant plusieurs mois pour les femmes des pays scandinaves. Forts de la connaissance de cette pratique chez les sociétés actuelles occidentales ou traditionnelles, les enjeux en matière d'allaitement du passé seraient d'analyser la relation entre ces pratiques et:

- les paramètres biologiques comme le sexe des enfants, ou l'état sanitaire déduit des lésions osseuses et dentaires;

- les paramètres paléodémographiques ou encore;

- les données archéologiques comme le statut social inféré à partir du mobilier funéraire afin d'affiner l'identification des facteurs qui ont pu modeler le comportement des mères envers leurs nourrissons et leurs jeunes enfants.

\section{Déclaration d'intérêts}

L'auteur n'a pas transmis de déclaration de conflits d'intérêts.

\footnotetext{
10 Il s'agit notamment des indicateurs de stress, comme les hypoplasies de l'émail dentaire, les caries, la croissance en longueur. Ces marqueurs sont sensibles à l'environnement et aux modes de vie dans le passé.
}

\section{Références}

[1] Amir L. Social theory and Infant feeding. Int Breastfeed J 2011;6(7), http://dx.doi.org/10.1186/1746-4358-1186-1187.

[2] Dettwyler KA, Fishman C. Infant feeding practices and growth. Annu Rev Anthropol 1992;21:171-204.

[3] Herring DA, Saunders SR, Katzenberg MA. Investigating the weaning process in past populations. Am J of Phys Anthropol 1998;105(4):425-39.

[4] Flandrin JL. Le sexe et l'Occident. Évolution des attitudes et des comportements 1981:376p.

[5] Dupâquier J. Histoire de la population française. 1/Des origines à la Renaissance. Paris: Quadrige/Presses Universitaires Françaises; 1995, 559 p.

[6] Stuart-Macadam P. Breastfeeding in Prehistory. In: StuartMacadam P, Dettwyler KA, editors. Breastfeeding: biocultural perspectives. Hawthorne, New-York: Aldine de Gruyter; 1995. p. 75-99.

[7] Bonnet D, Le Grand-Sébille C, Morel MF. Allaitement en marge. Paris: L'Harmattan; 2002, $243 \mathrm{p}$.

[8] Thorvaldsen G. Was there a European breastfeeding pattern? The History of the Family 2008;13:283-95.

[9] Ibanez G, Martin N, Denantes M, Saurel-Cubizolles MJ, Ringa $\mathrm{V}$, Magnier AM. Prevelence of breastfeeding in industrialized countries. Rev Epidemiol Sante 2012;60:305-20.

[10] WHO. Expert consultation on the optimal duration of exclusive breastfeeding. Report of an expert consultation. World Health Organization, Geneva. 2001, p 10.

[11] Kennedy GE. From the ape's dilemna to the weanling's dilemna: early weanning and its evolutionnary context. J Hum Evol 2005;48:123-45.

[12] Sellen D. Comparison of infant feeding patterns reported for nonindustrial populations with current recommendations. J Nutr 2001;3:2707-15.

[13] Charbonneau H, Desjardins B, Guillemette A, Landry Y, Légaré J, Nault F. Naissance d'une population. Les Français établis au Canada au xvIl ${ }^{\mathrm{e}}$ siècle. Paris: INED, Presses de l'Université de Montréal; 1987, 249 p.

[14] Pison G, Hill K, Cohen B, Foote K. Les changements démographiques au Sénégal. Paris: INED, Collection PUF; 1997, 251 p.

[15] Flandrin J-L. L'attitude à l'égard du petit enfant et les conduites sexuelles dans la civilisation occidentale. Annales de Démographie Historique Édition Mouton: Paris (Structures anciennes et civilisation); 1973, 143-210.

[16] Morel M-F. Histoire de l'enfant dans la France ancienne (xv1 ${ }^{\mathrm{e}}-\mathrm{xIx} \mathrm{e}^{\mathrm{e}}$ siècle). Etudes Freudiennes 1995;36:244.

[17] Sellen D, Smay D. Relationship between subsistence and age at weaning in "preindustrial" societies. Human Nature 2001;12:47-87.

[18] Katzenberg A, Saunders RS. Biological Anthropology of the Human Skeleton. Hoboken, New Jersey: John Wiley \& Sons Inc - 2nd Revision edition; 2008, $680 \mathrm{p}$.

[19] van der Merwe NJ, Vogel JC. ${ }^{13} \mathrm{C}$ content of human collagen as a measure of prehistoric diet in Woodland North America. Nature 1978;276:815-6.

[20] Bocherens H, Fizet $M$, Mariotti A, Lange-Badre B, Vandermeersch B, Borel JP, et al. Isotopic biogeochemistry $\left({ }^{13} \mathrm{C},{ }^{15} \mathrm{~N}\right)$ of fossil vertebrate collag. J Hum Evol 1991;20:481-92.

[21] Herrscher E, Bocherens H, Valentin F, Colardelle R. Comportement alimentaire au Moyen Âge à Grenoble: application de la biogéochimie isotopique à la nécropole de Saint-Laurent ( $\mathrm{xIII}{ }^{\mathrm{e}}-\mathrm{xv}^{\mathrm{e}}$ siècles, Isère, France). Comptes rendus de l'Académie des sciences Série 2. Sciences de la Vie 2001;324:479-87.

[22] Prowse T, Schwarcz HP, Saunders S, Macchiarelli R, Bondioli L. Isotopic paleodiet studies of skeletons from the Imperial Roman-age cemetery of Isola Sacra, Rome, Italy. J Archaeol Sci 2004;31(3):259-72.

[23] Herrscher E, Le Bras-Goude G. Southern French Neolithic populations: isotopic evidence for regional specificities in environment and diet. Am J Phys Anthropol 2010;141:259-72.

[24] Valentin F, Buckley H, Herrscher E, Kinaston R, Bedford $S$, Spriggs $M$, et al. Lapita subsistence strategies and food 
consumption patterns in the community of Teouma (Efate Vanuatu). J Archaeol Sci 2010;37:1820-9.

[25] Fogel ML, Tuross N, Owsley DW. Nitrogen isotope tracers of human lactation in modern and archaeological populations. Annual Report of Geophysical Laboratory Carnegie Institution of Washington 1989:111-7.

[26] Katzenberg MA. Age differences and population variation in stable isotope values from Ontario, Canada. In: Lambert JB, Grupe G, editors. Prehistoric human bone Archaeology at the molecular level. 1993. p. 39-62.

[27] Katzenberg A, Herring A, Saunders SR. Weaning and infant mortality: evaluating the skeletal evidence. Yearbook Phys Anthropol 1996;39:177-99.

[28] Flandrin JL, Montanari M. Histoire de l'alimentation. Paris: Fayard; 1996, $915 \mathrm{p}$.

[29] Aubaile F, Bernard M, Pasquet P. La viande, un aliment, des symboles. Aix-en-Provence: Collection Écologie Humaine, Éditions Edisud; 2004, 247 p.

[30] Rollet C, Morel MF. Des bébés et des hommes. Traditions et modernité des soins aux tout-petits. Paris: Albin Michel; 2000, $385 \mathrm{p}$.

[31] Thoueille E. Lait et mythologie. In: Blin D, Thoueille E, Soulé $M$, editors. L'allaitement maternel: Une dynamique à bien comprendre. Toulouse: ERES; 2003. p. 35-42.

[32] Fildes V. The culture and biology of breastfeeding: An historical review of Western Europe. In: Stuart-Macadam P, Dettwyler KA, editors. Breastfeeding: biocultural perspectives. New-York: Aldine de Gruyter, Hawthorne; 1995. p. 75-99.

[33] Sussman GD. Selling Mothers' Milk. USA: University of Illinois Press; 1982, 210 p.

[34] Schwarz E, Ray R, Stuebe A, Allison M, Ness R, Freiberg M, et al. Duration of lactation and risk factors for maternal cardiovascular disease. Obstetr Gynecol 2009;113(5):974-82.

[35] Bartick M, Reinhold A. The burden of suboptimal breastfeeding in the United States: a pediatric cost analysis. Pediatrics 2010;125(5):1048-56.

[36] Robinson S, Fall C. Infant nutrition and later health: a review of current evidence. Nutrients 2012;4:859-74.

[37] Dykes F, Flacking R. Encouraging breastfeeding: a relational perspective. Early Human Develop 2010;86:733-6

[38] Katzenberg MA, Saunders SR, Fitzgerald WR. Age differences in stable carbon and nitrogen isotope ratios in a population of prehistoric maize horticulturists. Am J Phys Anthropol 1993;90(3):267-81.

[39] Dupras TL, Schwarcz HP, Fairgrieve SI. Infant feeding and weaning practices in Roman Egypt. Am J Phys Anthropol 2001;115:204-12.

[40] Wright LE, Schwarcz HP. Stable carbon and oxygen isotopes in human tooth enamel: identifying breastfeeding and weaning in Prehistory. Am J Phys Anthropol 1998;106(3): 411.

[41] Wright LE, Schwarcz HP. Correspondence between stable carbon, oxygen and nitogen isotopes in human tooth enamel and dentine: infant diets at Kaminaljuyù. J Archaeol Sci 1999;26(9):1159-70.

[42] Sillen A, Smith P. Weaning patterns are reflected in strontiumcalcium ratios of juvenile skeletons. J of Archaeological Sci 1984;11(3):237-45.

[43] DeNiro MJ, Epstein S. Influence of diet on the distribution of nitrogen isotopes in animals. Geochim Cosmochim Acta 1981;45:341-51.

[44] Schoeninger MJ, DeNiro MJ. Nitrogen and carbon isotopic composition of bone collagen from marine and terrestrial animals. Geochim Cosmochim Acta 1984;48:625-39.

[45] Bocherens H, Drucker D. Trophic level isotopic enrichment of carbon and nitrogen in bone collag case studies from recent ancient terrestrial ecosystems. Int J Osteoarchaeol 2003;13:46-53.

[46] Balter V, Simon L, Fouillet H, Lécuyer C. Box-modelling of ${ }^{15} \mathrm{~N} /{ }^{14} \mathrm{~N}$ in mammals. Oecologia 2005;147(2):212-22.

[47] Fuller BT, Fuller JL, Harris DA, Hedges REM. Detection of breastfeeding and weaning in modern human infants with carbon and nitrogen stable isotope ratios. Am J Phys Anthropol 2006;129(2):279-93.

[48] Polet C, Orban R. Dents et ossements humains: que mangeaiton au Moyen Âge. Belgique: Brepols; 2001, 173 p.

[49] Buikstra J, Konigsberg L, Bullington J. Fertility and the development of agriculture in the Prehistoric Midwest. Am Antiquity 1986;51:528-46

[50] Schurr MR, Powell ML. The role of changing childhood diets in the prehistoric evolution of food production: an isotopic assessment. Am J Phys Anthropol 2005;126(3):278-94.

[51] Schurr MR. Stable nitrogen isotopes as evidence for the age of weaning at the Angel site: comparison of isotopic and demographic measures of weaning age. J Archaeol Sci 1997;24:919-27.

[52] Schurr MR. Using stable nitrogen-isotopes to study weaning behavior in past populations. World Archaeol 1998;30: 327-42.

[53] Herrscher E. Alimentation d'une population historique. Analyse des données isotopiques de la nécropole Saint-Laurent de Grenoble $\left(\mathrm{xIII}^{\mathrm{e}}-\mathrm{xV}^{\mathrm{e}}\right.$ siècle, France). Bull Mem Soc d'Anthropol de Paris 2003;15(3-4):149-269.

[54] Pearson JA, Hedges REM, Molleson TI, Özbek M. Exploring the relationship between weaning and infant mortality: an isotope case study from Așıkl Höyük and Cayönü Tepesi. Am J Phys Anthropol 2010;143(3):448-57.

[55] Fuller BT, Molleson TI, Harris DA, Gilmour LT, Hedges REM. Isotopic evidence for breastfeeding and possible adult dietary differences from late/sub-roman Britain. Am J Phys Anthropol 2006;129(1):45-54.

[56] Prowse TL, Saunders SR, Schwarcz HP, Garnsey P, Macchiarelli $\mathrm{R}$, Bondioli L. Isotopic and dental evidence for infant and young child feeding practices in an imperial Roman skeletal sample. Am J Phys Anthropol 2008;137(3):294-308.

[57] Mays S, Richards MP, Fuller B. Bone stable isotope evidence for infant feeding in Mediaeval England. Antiquity 2002;76(293):654-6.

[58] Richards MP, Mays S, Fuller BT. Stable carbon and nitrogen isotope values of bone and teeth reflect weaning age at the Medieval Wharram Percy site, Yorkshire, UK. Am J Phys Anthropol 2002;119(3):205-10.

[59] Fuller BT, Richards MP, Mays SA. Stable carbon and nitrogen isotope variations in tooth dentine serial sections from Wharram Percy. J Archaeol Sci 2003;30:1673-84.

[60] Williams JS, White CD, Longstaffe FJ. Trophic level and macronutrient shift effects associated with the weaning process in the postclassic Maya. Am J Phys Anthropol 2005;128(4):781-90

[61] Smith BN, Epstein S. Two categories of ${ }^{13} \mathrm{C} /{ }^{12} \mathrm{C}$ ratios for higher plants. Plant Physiol 1971;47:380-4.

[62] DeNiro MJ, Epstein S. Influence of diet on the distribution of carbon isotopes in animals. Geochim Cosmochim Acta 1978;42:495-506.

[63] Deines P. The isotopic composition of reduced organic carbon. In: Fritz P, Fontes JC, editors. Handbook of environmental isotope geochemistry. New-York: Elsevier; 1980. p. 329-406.

[64] Ambrose SH, Norr L. Experimental evidence for the relationship of the carbon isotope ratios of whole diet and dietary protein to those of bone collagen and carbonate. In: Lambert JB, Grupe G, editors. Prehistoric human bone Archaeology at the molecular level. Berl: Springer-Verlag; 1993. p. 1-37.

[65] Sillen A, Kavanagh M. Strontium palaeodietary research: a review. Yearbook of Phys Anthropol 1982;25:67-90.

[66] Balasse M, Bocherens H, Tresset A, Mariotti A, Vigne JD. Emergence de la production laitière au Néolithique? Contribution de l'analyse isotopique d'ossements de bovins archéologiques. Comptes rendus de l'Académie des sciences Série 2, Sciences de la terre et des planètes 1997;325:105-10.

[67] Balasse $M$, Bocherens $H$, Mariotti A. Intra-bone variability of collagen and apatite isotopic composition used as evidence of a change of diet. J Archaeol Sci 1999;26:593-8.

[68] Ubelaker $\mathrm{DH}$. The estimation of age at death from immature human bone. In: Thomas CC, editor. Age Markers in the Human Skeleton. Springfield: Illinois; 1989. p. 55-70. 
[69] Herrscher E. Comportements socioculturels liés à l'allaitement et au sevrage: le cas d'une population grenobloise sous l'Ancien Régime. Ann Fyssen 2005;20:46-66.

[70] Herrscher E, Valentin F, Coupain L. Analyse bioarchéologique des enfants découverts dans le Chœur. In: Brut C, Valentin F, Herrscher E, editors. Rapport final d'Opération, Etude Anthropologique. Paris: DHAAP; 2012. p. 25 p.

[71] Herrscher E. Inferring diet by stable isotope analysis: a case study from the French Alps. In: Carver M, editor. The Archaeology of Medieval Europe Vol 2: The Twelfth to Sixteenth Centuries AD. Aarhus. Cophenhagen: Aarhus University Press; 2011. p. 139-46.

[72] Price TD, Grupe G, Schröter P. Reconstruction of migration patterns in the Bell Beaker period by stable strontium isotope analysis. Appl Geochem 1994;9:413-7.

[73] Alexandre-Bidon D, Lett D. Les enfants au Moyen Âge. La vie Quotidienne. Paris: Éditions Hachette; 1997, 280 p.

[74] Fizet $M$. Biogéochimie isotopique $\left({ }^{13} \mathrm{C}\right.$ et $\left.{ }^{15} \mathrm{~N}\right)$ du collagène des vertébrés : contribution à l'étude du paleoécosystéme anthropique du Pléistocène supérieur (Marillac Charente) [Thèse de Doctorat]. Paris: Université Paris IV, 1992, 136 p.
[75] Waters-Rist AL, Bazaliiskii VI, Weber AW, Katzenberg MA. Infant and child diet in Neolithic hunter-fisher-gatherers from cisbaikal, Siberia: Intra-long bone stable nitrogen and carbon isotope ratios. Am J Phys Anthropol 2011;146(2):225-41.

[76] Harvey PH, Clutton-Brock TH. Life history variation in primates. Evolution 1985;39:559-81.

[77] Clayton F, Sealy J, Pfeiffer S. Weaning age among foragers at Matjes river rock shelter South Africa, from stable nitrogen and carbon isotope analyses. Am J Phys Anthropol 2006;129(2):311-7.

[78] Jaouen K, Balter V, Herrscher E, Lamboux A, Telouk P, Albarède $\mathrm{F}$. Fe and $\mathrm{Cu}$ stable isotopes in archeological human bones and their relationship to sex. Am J Phys Anthropol 2012;148(3):334-40.

[79] Reynard LM, Pearson JA, Henderson GM, Hedges REM. Calcium isotopes in juvenile milk-consumers. Archaeometry: 2012;DOI:10.1111/j.1475-4754.2012.00715.x.

[80] Fewtrell M, Morgan J, Duggan C, Gunnlaugsson G, Hibberd P, Lucas $\mathrm{A}$, et al. Optimal duration of exclusive breastfeeding: what is the evidence to support current recommendations? Am J Clinic Nut 2007;85(2):635S-8S. 